\begin{tabular}{lr}
$\frac{\text { C O L L O Q U I U M }}{199^{*}}$ & M A T H E M A T I C U M \\
\hline VoL. LXIII & FAsC. 1 \\
\hline
\end{tabular}

\title{
THE MAZUR INTERSECTION PROPERTY FOR FAMILIES OF \\ CLOSED BOUNDED CONVEX SETS IN BANACH SPACES
}

BY

PRADIPTA BANDYOPADHYA Y A (CALCUTTA)

Introduction. S. Mazur [10] was the first to consider the following smoothness property in normed linear spaces, called the Mazur Intersection Property (MIP), or, more briefly, the Property (I):

Every closed bounded convex set is the intersection of closed balls containing it.

Later, R. R. Phelps [11] provided a dual characterization of this property for finite-dimensional spaces. Nearly two decades later, Phelps' results were extended by J. R. Giles, D. A. Gregory and B. Sims [9] to general normed linear spaces. They also showed that in dual Banach spaces the MIP implies reflexivity, and considered the weaker property that every weak* compact convex set in a dual space is the intersection of balls (Property weak*-I).

Subsequently, there appeared several papers dealing with similar intersection properties for compact convex sets [14, 12], weakly compact convex sets [16] and compact convex sets with finite affine dimension [13].

In the present work, we give a unified treatment of the intersection properties for these diverse classes of sets by considering the MIP for the members of a general family of closed bounded convex sets in a Banach space, and show that all the known results follow as special cases of our result. We also introduce a new condition of separation of convex sets which turns out to be equivalent to the intersection property in all known cases. This strengthens the results of Zizler [15]. As another application of our result, we extend our previous work [1] on lifting the MIP from a Banach space $X$ to the Lebesgue-Bochner space $L^{p}(\mu, X)(1<p<\infty)$.

We should point out that our proofs are usually modifications, refinements and adaptations to our very general set-up of arguments for particular cases to be found in [9], [12] and [14].

1991 Mathematics Subject Classification: Primary 46B20.

Key words and phrases: Mazur Intersection Property, duality map, support mapping, points of continuity, ( $\left.\mathrm{w}^{*}-\right)$ denting points, norming subspaces, Bochner $L^{p}$-spaces. 
Notations. We work only with real Banach spaces. The closed unit ball and the unit sphere of a Banach space $X$ will be denoted by $B(X)$ and $S(X)$ respectively. For $z \in X$ and $r>0$, we denote by $B_{r}[z]$ (resp. $\left.B_{r}(z)\right)$ the closed (resp. open) ball of radius $r$ and centre $z$. For $x \in S(X)$, $D(x)=\left\{f \in S\left(X^{*}\right): f(x)=1\right\}$. The set-valued map $D$ is called the duality map and any selection of $D$ is called a support mapping. For $K \subseteq X, f \in X^{*}$ and $\alpha>0$, the set $S(K, f, \alpha)=\{x \in K: f(x)>\sup f(K)-\alpha\}$ is called the open slice of $K$ determined by $f$ and $\alpha$. For $A \subseteq X$, denote by $\operatorname{co}(A)$ (resp. $\operatorname{aco}(A))$ the convex (resp. absolutely convex) hull of $A$. For $A \subseteq X$, $f \in X^{*},\|f\|_{A}=\sup \{|f(x)|: x \in A\}, A^{\circ}=\left\{f \in X^{*}:\|f\|_{A} \leq 1\right\}$ and for $B \subseteq X^{*}, A-\operatorname{dia}(B)=\sup \left\{\left\|b_{1}-b_{2}\right\|_{A}: b_{1}, b_{2} \in B\right\}$. For $A_{1}, A_{2} \subseteq X$, $\operatorname{dist}\left(A_{1}, A_{2}\right)=\inf \left\{\left\|x_{1}-x_{2}\right\|: x_{i} \in A_{i}, i=1,2\right\}$. For $A \subseteq X, \bar{A}^{\sigma}$ denotes the closure of $A$ for the topology $\sigma$. Whenever the topology is not specified, we mean the norm topology. We identify an element $x \in X$ with its canonical image $\widehat{x}$ in $X^{* *}$.

1. The set-up and main result. Let $X$ be a real Banach space, let $F$ be a closed norming subspace of $X^{*}$ (i.e., $\|\widehat{x}\|_{B(F)}=\|x\|$, for all $x \in X$ ), and let $\mathcal{C}$ be a family of norm bounded, $\sigma(X, F)$-closed convex sets with the following properties:

(A1) $C \in \mathcal{C}, x \in X$ and $\alpha \in \mathbb{R} \Rightarrow \alpha C+x \in \mathcal{C}$,

(A2) $C_{1}, C_{2} \in \mathcal{C} \Rightarrow \overline{\operatorname{aco}}^{\sigma(X, F)}\left(C_{1} \cup C_{2}\right) \in \mathcal{C}$,

(A3) $C \in \mathcal{C}, C$ absolutely convex and $f \in F \Rightarrow C \cap f^{-1}(0) \in \mathcal{C}$.

Note that (A1) implies that $\mathcal{C}$ contains all singletons.

EXAmples. (i) $\mathcal{C}=\{$ all closed bounded convex sets in $X\}, F=X^{*}$.

(ii) $X=Y^{*}, \mathcal{C}=\{$ all w*-compact convex sets in $X\}, F=\widehat{Y}$.

(iii) $\mathcal{C}=\{$ all compact convex sets in $X\}, F=$ any norming subspace.

(iv) $\mathcal{C}=\{$ all compact convex sets in $X$ with finite affine dimension $\}$, $F=$ any norming subspace.

(v) $\mathcal{C}=\{$ all weakly compact convex sets in $X\}, F=$ any norming subspace.

Let $\mathcal{F}=\left\{C^{\circ}: C \in \mathcal{C}\right\}$. Then $\mathcal{F}$ is a local base for a locally convex Hausdorff vector topology $\tau$ on $X^{*}$, the topology of uniform convergence on elements of $\mathcal{C}$. Clearly, $\tau$ is stronger than the $\mathrm{w}^{*}$-topology on $X^{*}$ and weaker than the norm topology.

Definitions. (1) Denote by $E_{\tau}$ the set of all extreme points of $B\left(X^{*}\right)$ which are points of continuity of the identity map id $:\left(B\left(X^{*}\right), \mathrm{w}^{*}\right) \rightarrow$ $\left(B\left(X^{*}\right), \tau\right)$. 
(2) For $C \in \mathcal{C}, C$ absolutely convex and $\varepsilon>0$, we say that a point $x \in S(X)$ belongs to the set $M_{C, \varepsilon}$ if there is a $\delta>0$ such that

$$
\sup _{y \in C, 0<\lambda<\delta} \frac{\|x+\lambda y\|+\|x-\lambda y\|-2}{\lambda}<\varepsilon .
$$

(3) $H_{\tau}=\bigcap\left\{{\overline{D\left(M_{C, \varepsilon}\right)}}^{\tau}: C \in \mathcal{C}, C\right.$ absolutely convex, $C \subseteq B(X)$ and $\varepsilon>0\}$.

Lemma 1. For any absolutely convex $C \in \mathcal{C}, C \subseteq B(X), x \in S(X)$ and $\varepsilon>0$, the following are equivalent:

(i) $x \in M_{C, \varepsilon}$.

(ii) There is a $\delta \in(0,1)$ such that $C$-dia $S\left(B\left(X^{*}\right), \widehat{x}, \delta\right)<\varepsilon$.

(iii) There is a $\delta \in(0,1)$ such that $C$-dia $\left[\bigcup\left\{D(y): y \in S(X) \cap B_{\delta}(x)\right\}\right]$ $<\varepsilon$.

Proof. We omit the proof, which is an easy modification of the proof of Lemma 2.1 in [9].

Now we have our main result:

THEOREM 1. If $X, F$ and $\mathcal{C}$ are as above, consider the following statements:

(a) $F \subseteq{\overline{\mathbb{R}}+E_{\tau}}^{\tau}$.

(b) $F \subseteq{\overline{\mathbb{R}^{+} H_{\tau}}}^{\tau}$.

(c) If $C_{1}, C_{2} \in \mathcal{C}$ are such that there exists $f \in F$ with $\sup f\left(C_{1}\right)<$ inf $f\left(C_{2}\right)$ then there exist disjoint closed balls $B_{1}, B_{2}$ such that $C_{i} \subseteq B_{i}$, $i=1,2$.

(d) Every $C \in \mathcal{C}$ is the intersection of closed balls containing it.

(e) For every norm dense subset $A$ of $S(X)$ and every support mapping $\phi: S(X) \rightarrow S\left(X^{*}\right), F \subseteq{\overline{\mathbb{R}^{+} \phi(A)}}^{\tau}$.

Then we have $(\mathrm{a}) \Rightarrow(\mathrm{b}) \Rightarrow(\mathrm{c}) \Rightarrow(\mathrm{d}) \Rightarrow(\mathrm{e})$.

(For the converse implications, see corollaries and remarks at the end of this section.)

Proof. $(\mathrm{a}) \Rightarrow(\mathrm{b})$. It is enough to prove $E_{\tau} \subseteq H_{\tau}$.

Let $f \in E_{\tau}$. Let $C \in \mathcal{C}, C$ absolutely convex, $C \subseteq B(X)$ and $\varepsilon>0$. We want to prove $f \in{\overline{D\left(M_{C, \varepsilon}\right)}}^{\tau}$. Let $K \in \mathcal{C}$ and $0<\eta<\varepsilon$. We may assume $K \subseteq B(X)$. Let $K_{0}=\overline{\mathrm{aco}}^{\sigma(X, F)}(K \cup C)$. Then $K_{0} \subseteq B(X)$ as $B(X)$ is $\sigma(X, F)$-closed. Note that $K_{0} \in \mathcal{C}$ by $(\mathrm{A} 2)$.

Since $f$ is an extreme point of the $\mathrm{w}^{*}$-compact convex set $B\left(X^{*}\right)$ and id : $\left(B\left(X^{*}\right), \mathrm{w}^{*}\right) \rightarrow\left(B\left(X^{*}\right), \tau\right)$ is continuous at $f$, it follows from the theorem on p. 107 of [5] that the $\mathrm{w}^{*}$-slices of $B\left(X^{*}\right)$ containing $f$ form a base for 
the relative $\tau$-topology at $f$. Thus there exist $x \in S(X)$ and $0<\delta<1$ such that $f \in S=S\left(B\left(X^{*}\right), \widehat{x}, \delta\right)$ and $K_{0}-\operatorname{dia}(S)<\eta$.

Now, by Lemma $1, x \in M_{K_{0}, \eta} \subseteq M_{C, \varepsilon}$ and for any $f_{x} \in D(x), f_{x} \in$ $D\left(M_{C, \varepsilon}\right)$ and $f_{x} \in S$, so $\left\|f-f_{x}\right\|_{K} \leq\left\|f-f_{x}\right\|_{K_{0}}<\eta$.

(b) $\Rightarrow(\mathrm{c})$. Let $C_{1}, C_{2} \in \mathcal{C}$ and $f \in S(F)$ be such that $\sup f\left(C_{1}\right)<$ $\inf f\left(C_{2}\right)$. Let $z \in X$ be such that $f(z)=\frac{1}{2}\left(\sup f\left(C_{1}\right)+\inf f\left(C_{2}\right)\right)$ and put $\varepsilon=\frac{1}{12}\left(\inf f\left(C_{2}\right)-\sup f\left(C_{1}\right)\right)>0$. Then $\inf f\left(C_{2}-z\right)>5 \varepsilon$ and $\inf (-f)\left(C_{1}-z\right)>5 \varepsilon$. We may assume without loss of generality that $z=0, C_{i} \subseteq B(X), i=1,2$, and $\|f\|=1$. Let $K=\overline{\operatorname{aco}}^{\sigma(X, F)}\left(C_{1} \cup C_{2}\right)$; then $K \in \mathcal{C}, K$ is absolutely convex and $K \subseteq B(X)$.

By (b), there are $\lambda \geq 0$ and $g \in H_{\tau}$ such that $\|f-\lambda g\|_{K}<\varepsilon$. If $\lambda=0$, we have $\|f\|_{K}<\varepsilon$, and hence $\inf _{C_{2}} f<\varepsilon$, a contradiction. Thus, $\lambda>0$.

Now, $g \in H_{\tau} \subseteq{\overline{D\left(M_{K, \varepsilon / \lambda}\right.}}^{\tau}$. So, we can find $x \in M_{K, \varepsilon / \lambda}$ and $h \in D(x)$ such that $\|g-h\|_{K}<\varepsilon / \lambda$. By definition, there is a $\delta>0$ such that

$$
\sup _{y \in K, 0<\alpha<\delta} \frac{\|x+\alpha y\|+\|x-\alpha y\|-2}{\alpha}<\frac{\varepsilon}{\lambda} .
$$

Choose an integer $n>\lambda /(\varepsilon \delta)$. The proof will be complete once we show that $B_{1}=B_{(n-1) \varepsilon / \lambda}[-n \varepsilon x / \lambda]$ and $B_{2}=B_{(n-1) \varepsilon / \lambda}[n \varepsilon x / \lambda]$ work.

Clearly, $B_{1}$ and $B_{2}$ are disjoint. Suppose, if possible, $y \in C_{2}$ and $y \notin B_{2}$. Then $y \in K$. Take $\alpha=\lambda /(n \varepsilon)<\delta$ and observe that

$$
\begin{aligned}
\frac{\|x+\alpha y\|+\|x-\alpha y\|-2}{\alpha}=\frac{\|x+\alpha y\|-\|x\|}{\alpha}+\left\|\frac{x}{\alpha}-y\right\|-\frac{1}{\alpha} \\
\geq h(y)+\frac{(n-1) \varepsilon}{\lambda}-\frac{n \varepsilon}{\lambda}=h(y)-\frac{\varepsilon}{\lambda} \geq g(y)-\frac{2 \varepsilon}{\lambda} \\
\quad=\frac{1}{\lambda}[\lambda g(y)-2 \varepsilon]>\frac{1}{\lambda}[f(y)-3 \varepsilon] \geq \frac{1}{\lambda}[5 \varepsilon-3 \varepsilon]=\frac{2 \varepsilon}{\lambda} .
\end{aligned}
$$

This contradicts the fact that $x \in M_{K, \varepsilon / \lambda}$. The other inclusion follows similarly once we note that $K$, and hence $M_{K, \varepsilon / \lambda}$, is symmetric and $h \in D(x)$ implies $(-h) \in D(-x)$.

(c) $\Rightarrow(d)$. Since singletons are in $\mathcal{C}$ and every $C \in \mathcal{C}$ is $\sigma(X, F)$-closed, (d) follows from (c).

(d) $\Rightarrow($ e). (We adapt Phelps' [11] arguments.) Let $A$ be a norm dense subset of $S(X)$ and let $\phi$ be a support mapping. Let $f \in S(F), K \in \mathcal{C}$ and $0<\varepsilon<1$. We may assume $K \subseteq B(X)$ and further that $K$ is absolutely convex and $\|f\|_{K}>1-\varepsilon / 2$. (Let $x \in B(X)$ be such that $f(x)>1-\varepsilon / 2$. Let $L=\overline{\operatorname{aco}}^{\sigma(X, F)}[\{x\} \cup K]$. Then $L \subseteq B(X), L \in \mathcal{C}$ and $\|\cdot\|_{L} \geq\|\cdot\|_{K}$.) Let $u \in K$ be such that $f(u)>1-\varepsilon / 2$. Put $u^{\prime}=\frac{1}{4} \varepsilon u$ and $D=K \cap f^{-1}(0)$. Then $D \in \mathcal{C}$ [by (A3)] and $u^{\prime} \notin D$. By (c), there exist $z \in X$ and $r>0$ such that $D \subseteq B_{r}[z]$ and $\left\|u^{\prime}-z\right\|>r$. 
Let $\mu=\left\|u^{\prime}-z\right\|-r>0$. Put

$$
w=\frac{1}{r+\mu}\left(r u^{\prime}+\mu z\right) .
$$

Then $\|w-z\|=r$. Put $x=(1 / r)(w-z) \in S(X)$. Let $C=\overline{\operatorname{co}}\left[\left\{u^{\prime}\right\} \cup B_{r}[z]\right]$. Let $0<\delta<\mu /(r+\mu)$. If $p \in B_{r \delta}[w]$, then $\|p-w\|<r \mu /(r+\mu)$, so

$$
p=w+\frac{r \mu}{r+\mu} \cdot y
$$

for some $y \in X,\|y\|<1$. Thus,

$$
p=\frac{r}{r+\mu} u^{\prime}+\frac{\mu}{r+\mu}(z+r y) .
$$

Now, $z+r y \in B_{r}(z)$, and hence $p \in \operatorname{Int}(C)$, the interior of $C$. So, $B_{r \delta}[w] \subseteq$ $\operatorname{Int}(C)$.

Let $y \in B_{\delta}[x] \cap A$ and $g=\phi(y)$. Put $v=r y+z$. Clearly, $\|v-w\| \leq r \delta$, hence $v \in \operatorname{Int}(C)$ and $g(v)=\sup g\left(B_{r}[z]\right)$. Now, $v \in \operatorname{Int}(C) \Rightarrow$ there exists $t \in(0,1)$ and $v^{\prime} \in B_{r}(z)$ such that $v=t u^{\prime}+(1-t) v^{\prime}$. Thus, $g(v)=\operatorname{tg}\left(u^{\prime}\right)+(1-t) g\left(v^{\prime}\right)<\operatorname{tg}\left(u^{\prime}\right)+(1-t) g(v)$. Also, $0 \in D \subseteq B_{r}[z] \Rightarrow$ $0 \leq g(v)<g\left(u^{\prime}\right)=\frac{1}{4} \varepsilon g(u) \leq \frac{1}{4} \varepsilon\|g\|_{K}$. So, $0<\|g\|_{K} \leq\|g\|=1$. Put $\lambda=1 /\|g\|_{K}$. Then $\sup \lambda g(D) \leq \sup \lambda g\left(B_{r}[z]\right)=\lambda g(v)<\frac{1}{4} \varepsilon\|\lambda g\|_{K}=\frac{1}{4} \varepsilon$. By symmetry of $D,\|\lambda g\|_{D} \leq \frac{1}{4} \varepsilon$.

Now, by Phelps' Lemma [11, Lemma 3.1] applied to the linear space $\operatorname{sp}(K)$ spanned by $K$, equipped with $\mu_{K}$, the gauge or Minkowski functional of $K$, we have

$$
\left\|\frac{f}{\|f\|_{K}}+\lambda g\right\|_{K} \leq \frac{\varepsilon}{2} \quad \text { or } \quad\left\|\frac{f}{\|f\|_{K}}-\lambda g\right\|_{K} \leq \frac{\varepsilon}{2} .
$$

But $u \in K$ and $\varepsilon<1$ implies $f(u) /\|f\|_{K} \geq f(u)>1-\varepsilon / 2>\varepsilon / 2$ and $\lambda g(u)>0$. Thus, $\|f /\| f\left\|_{K}-\lambda g\right\|_{K} \leq \varepsilon / 2$. Then we have

$$
\|f-\lambda g\|_{K} \leq \frac{\varepsilon}{2}+\left\|\frac{f}{\|f\|_{K}}-f\right\|_{K}=\frac{\varepsilon}{2}+\left(1-\|f\|_{K}\right) \leq \frac{\varepsilon}{2}+\frac{\varepsilon}{2}=\varepsilon .
$$

Corollary 1. If in the set-up of Theorem 1 , the set $A=\{x \in S(X)$ : $\left.D(x) \cap E_{\tau} \neq \emptyset\right\}$ is norm dense in $S(X)$, then all the statements in Theorem 1 are equivalent.

Proof. We simply note that in this case there is a support mapping that maps $A$ into $E_{\tau}$, and hence (e) $\Rightarrow(\mathrm{a})$.

Corollary $2[13,14,12]$. In the case of examples (iii), i.e., $\mathcal{C}=$ $\{$ all compact convex sets in $X\}, F=$ any norming subspace, and (iv), i.e., $\mathcal{C}=\{$ all compact convex sets in $X$ with finite affine dimension $\}, F=$ any norming subspace, all the statements in Theorem 1 are equivalent and (c) can be reformulated as 
$\left(\mathrm{c}^{\prime}\right)$ Disjoint members of $\mathcal{C}$ can be separated by disjoint closed balls.

Proof. In example (iii), $\tau$ is the bw* topology (see [7] for more on bw* topology) and in example (iv), $\tau$ is the $\mathrm{w}^{*}$-topology on $X^{*}$ and in both cases $\bar{F}^{\tau}=X^{*}$, so we may as well take $F=X^{*}$. Further, as the bw* topology agrees with the $\mathrm{w}^{*}$-topology on bounded sets, in both the cases $E_{\tau}=$ \{extreme points of $\left.B\left(X^{*}\right)\right\}$. Clearly, in both cases $A$ as in Corollary 1 is $S(X)$, and so in Theorem 1 all the statements are equivalent.

Since members of $\mathcal{C}$ in both cases are $\sigma(X, F)$-compact, $(\mathrm{c}) \Leftrightarrow\left(\mathrm{c}^{\prime}\right)$.

R e m arks. 1 . In example (v), i.e., $\mathcal{C}=\{$ all weakly compact convex sets in $X\}, F=$ any norming subspace, $\tau$ is the Mackey topology on $X^{*}$ (see [5] for further information), and again $\bar{F}^{\tau}=X^{*}$.

In this case, we do not know whether any of the implications in Theorem 1 can be reversed. However, we note that $(\mathrm{a}) \Rightarrow(\mathrm{d})$ in Theorem 1 gives a weaker sufficiency condition for MIP for weakly compact sets than the one used in [16]. And in this case, too, (c) and ( $\left.\mathrm{c}^{\prime}\right)$ of Corollary 2 are equivalent.

2. It seems unlikely that, in general, the implications in Theorem 1 can be reversed. Nevertheless, it appears to be an interesting and difficult problem to find conditions on $X, F$, and $\mathcal{C}$ under which this can be done.

However, there is yet another situation when the statements can actually be shown to be equivalent. And particular cases of this yield the characterizations of MIP and $\mathrm{w}^{*}$-MIP, i.e., examples (i) and (ii). This we take up in the next section.

3. Note that the subspace $F \subseteq X^{*}$ was assumed to be norming in order to ensure that the $\sigma(X, F)$-closure of norm bounded sets remains norm bounded, which is implicit in (A2). However, if (A2) is satisfied, as in examples (iii), (iv) and (v), for any total subspace $F$, our results easily carry through with only minor technical modifications in the proofs.

2. The MIP with respect to a norming subspace $F$. Our standing assumption in this section is that $F$ is a closed subspace of $X^{*}$ such that the set $T_{F}=\{x \in S(X): D(x) \cap S(F) \neq \emptyset\}$ (we shall write simply $T$ when there is no confusion) is a norm dense subset of $S(X)$. Then $F$ is necessarily norming. However, one can give examples (see below) of norming subspaces where this property does not hold. Let $\mathcal{C}=\{$ all norm bounded, $\sigma(X, F)$ closed convex sets in $X\}$. We say that $X$ has $F$-MIP if every $C \in \mathcal{C}$ is the intersection of closed balls containing it.

ExAmples. (i) $F=X^{*}, T=S(X)$ and we have the MIP.

(ii) $X=Y^{*}, F=\widehat{Y}, T=D(S(Y))$, which is dense by the Bishop-Phelps Theorem [2], and we have the $\mathrm{w}^{*}$-MIP.

Now, since $B(X) \in \mathcal{C}, \tau$ is the norm topology on $X^{*}$ and $E_{\tau}=\left\{\mathrm{w}^{*}\right.$ denting points of $B\left(X^{*}\right)$ \}. 
We need the following reformulation of Lemma 1:

LEMma 2. For $x \in S(X), F, T$ as above and $\varepsilon>0$, the following are equivalent:

(i) $x \in M_{\varepsilon}$.

(ii) $x$ determines a slice of $B(F)$ of diameter less than $\varepsilon$.

(iii) There exists $\delta>0$ such that

$$
\operatorname{dia}\left[\bigcup\left\{D(y) \cap S(F): y \in T \cap B_{\delta}(x)\right\}\right]<\varepsilon .
$$

Proof. (i) $\Rightarrow($ ii $) \Rightarrow$ (iii) follows again as easy adjustments of Lemma 2.1 in $[9]$.

(iii) $\Rightarrow\left(\right.$ i). Let $\delta>0$ be as in (iii). Let $d_{0}=\operatorname{dia}[\bigcup\{D(y) \cap S(F): y \in T \cap$ $\left.\left.B_{\delta}(x)\right\}\right]<\varepsilon$. Choose $\delta_{0}>0$ such that $\delta_{0}^{2}+2 \delta_{0}<\delta$ and $\delta_{0}^{2}+2 \delta_{0} / \varepsilon<1-d_{0} / \varepsilon$. Let $y \in S(X), 0<\lambda<\delta_{0}$. Then

$$
\begin{aligned}
\left\|\frac{x \pm \lambda y}{\|x \pm \lambda y\|}-x\right\| & \leq\left\|\frac{x \pm \lambda y}{\|x \pm \lambda y\|}-(x \pm \lambda y)\right\|+\lambda=|1-\|x \pm \lambda y\||+\lambda \\
& =|\|x\|-\|x \pm \lambda y\||+\lambda \leq 2 \lambda .
\end{aligned}
$$

Find $x_{1}, x_{2} \in T$ such that

$$
\left\|\frac{x+\lambda y}{\|x+\lambda y\|}-x_{1}\right\| \leq \lambda^{2} \text { and }\left\|\frac{x+\lambda y}{\|x+\lambda y\|}-x_{2}\right\| \leq \lambda^{2} .
$$

Let $f_{1}, f_{2} \in S(F)$ such that $f_{i} \in D\left(x_{i}\right), i=1,2$. Observe that $\left\|x_{i}-x\right\| \leq$ $\lambda^{2}+2 \lambda \leq \delta_{0}^{2}+2 \delta_{0}<\delta$, i.e., $x_{1}, x_{2} \in T \cap B_{\delta}(x)$. Thus $\left\|f_{1}-f_{2}\right\| \leq d_{0}$. Now,

$$
0 \leq 1-f_{1}\left(\frac{x+\lambda y}{\|x+\lambda y\|}\right)=f_{1}\left(x_{1}-\frac{x+\lambda y}{\|x+\lambda y\|}\right) \leq\left\|\frac{x+\lambda y}{\|x+\lambda y\|}-x_{1}\right\| \leq \lambda^{2} \text {. }
$$

So, $f_{1}(x+\lambda y) \geq\left(1-\lambda^{2}\right)\|x+\lambda y\|$. Similarly, $f_{2}(x-\lambda y) \geq\left(1-\lambda^{2}\right)\|x-\lambda y\|$. So, we have

$$
\begin{aligned}
\frac{\|x+\lambda y\|+\|x-\lambda y\|-2}{\lambda} & \leq \frac{f_{1}(x+\lambda y)+f_{2}(x-\lambda y)-2\left(1-\lambda^{2}\right)}{\lambda\left(1-\lambda^{2}\right)} \\
& =\frac{\left(f_{1}+f_{2}\right)(x)-2+\lambda\left(f_{1}-f_{2}\right)(y)+2 \lambda^{2}}{\lambda\left(1-\lambda^{2}\right)} \\
& \leq \frac{\left\|f_{1}-f_{2}\right\|+2 \lambda}{1-\lambda^{2}} \leq \frac{d_{0}+2 \lambda}{1-\lambda^{2}} \leq \frac{d_{0}+2 \delta_{0}}{1-\delta_{0}^{2}}
\end{aligned}
$$

(since $\frac{d_{0}+2 \lambda}{1-\lambda^{2}}$ is increasing in $\lambda$ ). Thus,

$$
\sup _{y \in S(X), 0<\lambda<\delta_{0}} \frac{\|x+\lambda y\|+\|x-\lambda y\|-2}{\lambda} \leq \frac{d_{0}+2 \delta_{0}}{1-\delta_{0}^{2}}<\varepsilon
$$

by the choice of $\delta_{0}$. 
R e mark. In [9], Lemma 3.1, this result was proved for $X=Y^{*}, F=\widehat{Y}$ using Bollobás' estimates for the Bishop-Phelps Theorem (see [2] and [3]). Specifically, the authors of [9] used the fact that in this case the following holds:

(*) For every $x \in S(X)$ and every sequence $\left\{f_{n}\right\} \subseteq S(F)$ such that $f_{n}(x) \rightarrow 1$, there exists a sequence $\left\{x_{n}\right\} \subseteq T$ and $f_{x_{n}} \in D\left(x_{n}\right) \cap S(F)$ such that $\left\|x_{n}-x\right\| \rightarrow 0$ and $\left\|f_{x_{n}}-f_{n}\right\| \rightarrow 0$.

In fact, one can show that in this situation, the following stronger property holds (see [8]):

(**) For every $x \in S(X), f \in S(F)$ and $\varepsilon>0$ with $f(x)>1-\varepsilon^{2}$ there exist $y \in T$ and $f_{y} \in D(y) \cap S(F)$ such that $\|x-y\| \leq \varepsilon$ and $\left\|f-f_{y}\right\| \leq \varepsilon$.

Using the fact that $(* *)$ holds for $F=X^{*}$, one can show that (**) also holds if $F$ is an L-summand in $X^{*}$, i.e., there is a projection $P$ on $X^{*}$ with $P\left(X^{*}\right)=F$ such that for any $f \in X^{*},\|f\|=\|P f\|+\|f-P f\|$. Clearly, if $(* *)$ holds for the pair $(X, F)$, it also holds for the pair $(F, \widehat{X})$. In particular, $(* *)$ holds for each of the following:

(1) $X=C[0,1], F=\{$ discrete measures on $[0,1]\}$,

(2) $X=C[0,1], F=\{$ absolutely continuous measures on $[0,1]\}$,

(3) $X=L^{1}[0,1], F=C[0,1]$.

So, in these cases, $(*)$ also holds and the proof of [9] can be used to prove Lemma 2.

However, one can construct examples (see below) to show that (**) does not, in general, follow from the density of $T$ in $S(X)$. It would be interesting to know whether $(*)$ does (in the absence of this information, we were forced to give a proof of (iii) $\Rightarrow$ (i) in Lemma 2 above which depended only on our standing assumption). Also, it would be interesting to find general sufficiency conditions for $(* *)$ to hold which would cover at least the case $X=Y^{*}$ and $F=\widehat{Y}$. In particular, is the following obviously necessary condition also sufficient for (**) to hold: $T$ is dense in $S(X)$ and $D(T) \cap S(F)$ is dense in $S(F)$ ? But these may be difficult problems.

Now, we are in a position to prove

THEOREM 2. If $X, F$ and $T$ are as above, the following are equivalent:

(a) The $w^{*}$-denting points of $B\left(X^{*}\right)$ are norm dense in $S(F)$.

(b) For every $\varepsilon>0, D\left(M_{\varepsilon}\right) \cap S(F)$ is norm dense in $S(F)$.

(c) If $C_{1}, C_{2} \in \mathcal{C}$ are such that there exists $f \in F$ with $\sup f\left(C_{1}\right)<$ $\inf f\left(C_{2}\right)$ then there exist disjoint closed balls $B_{1}, B_{2}$ such that $C_{i} \subseteq B_{i}$, $i=1,2$. 
(d) $X$ has the F-MIP.

(e) For every $f \in S(F)$ and $\varepsilon>0$, there exist $x \in T$ and $\delta>0$ such that $y \in B_{\delta}(x) \cap T$ implies $D(y) \cap S(F) \subseteq B_{\varepsilon}[f]$.

(f) For every support mapping $\phi$ that maps $T$ into $S(F)$ and every norm dense subset $A$ of $T, \phi(A)$ is norm dense in $S(F)$.

(Observe that since $F$ is norming, $\left\{\mathrm{w}^{*}\right.$-denting points of $\left.B\left(X^{*}\right)\right\} \subseteq S(F)$ and since $M_{\varepsilon}$ is open and $T$ is dense, $D\left(M_{\varepsilon}\right) \cap S(F)$ is non-empty whenever $M_{\varepsilon}$ is. Following the literature, the condition (e) may be called "quasicontinuity" of the set-valued map $D_{F}: T \rightarrow S(F)$ defined by $D_{F}(x)=$ $D(x) \cap S(F)$.)

Proof. $(\mathrm{a}) \Rightarrow(\mathrm{b})$. Let $f$ be a w*-denting point of $B\left(X^{*}\right)$ and $\varepsilon>0$. As noted above, $f \in S(F)$. Proceeding as in Theorem 1 (we may take $K=B(X)$ and so, $\left.K_{0}=B(X)\right)$, for any $0<\eta<\varepsilon$ there are $x \in S(X)$ and $\alpha>0$ such that $f \in S=S\left(B\left(X^{*}\right), \widehat{x}, \alpha\right)$ and $\operatorname{dia}(S)<\eta$. Since $T$ is dense in $S(X)$, by Lemma 1.1 of [9] there are $y \in T$ and $\delta>0$ such that $f \in S^{\prime}=S\left(B\left(X^{*}\right), \widehat{y}, \delta\right)$ and $S^{\prime} \subseteq S$. Again as in Theorem 1,y $\in M_{\varepsilon} \cap T$ and for any $f_{y} \in D(y) \cap S(F),\left\|f_{y}-f\right\|<\eta$.

$(\mathrm{b}) \Rightarrow(\mathrm{c}) \Rightarrow(\mathrm{d}) \Rightarrow(\mathrm{f})$. Just a simplified version of the implication (b) $\Rightarrow(\mathrm{c})$ $\Rightarrow(\mathrm{d}) \Rightarrow(\mathrm{e})$ in Theorem 1 where we replace $K$ by $B(X)$.

$(\mathrm{e}) \Leftrightarrow(\mathrm{f})$. An easy adjustment of the corresponding proof in Theorem 2.1 of $[9]$.

(e) $\Rightarrow(\mathrm{b})$. Let $f \in S(F)$ and $\varepsilon>0$. Let $0<\eta<\varepsilon / 2$. By (e), there exist $x \in T$ and $\delta>0$ such that $y \in T \cap B_{\delta}(x)$ implies $D(y) \cap S(F) \subseteq B_{\eta}[f]$. But then $\operatorname{dia}\left[\bigcup\left\{D(y) \cap S(F): y \in T \cap B_{\delta}(x)\right\}\right] \leq 2 \eta<\varepsilon$. So by Lemma 2, $x \in M_{\varepsilon} \cap T$ and $f_{x} \in D(x) \cap S(F)$ implies $\left\|f_{x}-f\right\|<\eta$.

(b) $\Rightarrow(\mathrm{a})$. For $n \geq 1$, let $D_{n}=\left\{f \in S(F): f\right.$ is contained in a $\mathrm{w}^{*}$-open slice of $B(F)$ of diameter $<1 / n\}$. By Lemma 2, $D\left(M_{1 / n}\right) \cap S(F) \subseteq D_{n}$. Thus, for all $n \geq 1, D_{n}$ is a norm open dense subset of $S(F)$ and by the Baire Category Theorem, $\bigcap D_{n}$ is norm dense in $S(F)$. But it is easy to see that $\bigcap D_{n}=\left\{\mathrm{w}^{*}\right.$-denting points of $\left.B\left(X^{*}\right)\right\}$.

Remarks. 1. Using techniques of [8], one can directly prove $(d) \Rightarrow(a)$.

2. The characterizations of MIP and $\mathrm{w}^{*}$-MIP (Theorems 2.1 and 3.1 of [9]) follow immediately from Theorem 2 , once we observe that $\{\widehat{x}: x$ is a denting point of $B(X)\}=\left\{\mathrm{w}^{*}\right.$-denting points of $\left.B\left(X^{* *}\right)\right\}$.

Corollary 3. (a) A real Banach space $X$ has the MIP if and only if whenever $C_{1}, C_{2}$ are closed bounded convex sets in $X$ with $\operatorname{dist}\left(C_{1}, C_{2}\right)>0$, there exist disjoint closed balls $B_{1}, B_{2}$ such that $C_{i} \subseteq B_{i}, i=1,2$.

(b) A dual Banach space $X^{*}$ has the $w^{*}-M I P$ if and only if disjoint $w^{*}$-compact convex sets in $X^{*}$ can be separated by disjoint closed balls.

Proof. (a) Let $\operatorname{dist}\left(C_{1}, C_{2}\right)=\delta>0$. Let $K_{2}=\overline{C_{2}+B_{\delta / 2}(0)}$. Then 
$C_{1}$ and $K_{2}$ are disjoint closed convex sets and $K_{2}$ has non-empty interior. Now, $f \in X^{*}$ that separates $C_{1}$ and $K_{2}$ strictly separates $C_{1}$ and $C_{2}$. Thus (a) follows.

The proof of (b) is immediate.

R e m a r k. Since disjoint closed balls always have positive distance, this corollary cannot be strengthened. Note that this corollary and Corollary 2 considerably strengthen the corollaries on p. 341 and p. 343 respectively of $[15]$.

ExAmple. Let $X$ be a non-reflexive Banach space. Let $F \subseteq X^{*}$ be a norming subspace which is an L-summand in $X^{*}$. Let $P$ be the corresponding L-projection. Let $f_{0} \in(I-P)\left(X^{*}\right)$ be such that $\left\|f_{0}\right\|=1$ and $f_{0}$ does not attain its norm on $B(X)$. Let $F_{1}=F \oplus_{1} \mathbb{R} f_{0}$. Then $F_{1}$ is a norming subspace of $X^{*}$ and $f_{0} \in S\left(F_{1}\right)$. Let $0<\varepsilon<1 / 2$. Suppose there exist $x \in S(X), g \in S\left(F_{1}\right)$ such that $\left\|f_{0}-g\right\|<\varepsilon$ and $g(x)=1$.

Now, $g=f+\alpha f_{0}$ for some $f \in F, \alpha \in \mathbb{R}$. We have $1=\|g\|=$ $\|f\|+\left\|\alpha f_{0}\right\|=\|f\|+|\alpha|$. If $\alpha=0, g=f$ and we have $\varepsilon>\left\|f_{0}-g\right\| \geq$ $\left\|P f_{0}-P g\right\|=\|g\|=1$. So, $\alpha \neq 0$. Also, $f=0$ implies $g=\alpha f_{0}$ and so $f_{0}(x)= \pm 1$, a contradiction, as $f_{0}$ does not attain its norm. Thus, $f \neq 0$.

But then

$$
1=g(x)=f(x)+\alpha f_{0}(x)=\|f\| \cdot \frac{f}{\|f\|}(x)+|\alpha| f_{0}\left(\frac{a x}{|\alpha|}\right) \leq\|f\|+|\alpha|=1
$$

This implies $f_{0}(a x /|\alpha|)=1$, again a contradiction.

As noted earlier, the pair $(X, F)$ satisfies $(* *)$, so $T_{F}$ is dense in $S(X)$ and $D\left(T_{F}\right) \cap S(F)$ is dense in $S(F)$. Now, clearly $T_{F_{1}} \supseteq T_{F}$, but the above shows that $D\left(T_{F_{1}} \cap S\left(F_{1}\right)\right.$ is not dense in $S\left(F_{1}\right)$. Consequently, though $T_{F_{1}}$ is dense in $S(X),(* *)$ is not satisfied.

Also, interchanging the roles of $X$ and $F_{1}$, the above shows that though $\widehat{X}$ is a norming subspace of $F_{1}^{*}, T_{\widehat{X}}=D\left(T_{F_{1}}\right) \cap S\left(F_{1}\right)$ is not dense, i.e., our standing assumption is not satisfied.

Finally, we note that $X=C[0,1], F=\{$ discrete measures on $[0,1]\}$ and $f_{0}=\left.\lambda\right|_{[0,1 / 2]}-\left.\lambda\right|_{[1 / 2,1]}$ satisfies the hypothesis of the above example, where $\left.\lambda\right|_{A}$ denotes the restriction of the Lebesgue measure $\lambda$ to the subset $A \subseteq[0,1]$.

3. An application to Bochner $L^{p}$-spaces. If $Z$ is a Banach space and $(\Omega, \Sigma, \mu)$ a measure space, let $L^{p}(\mu, Z)$ denote the Lebesgue-Bochner function space of $Z$-valued $p$-integrable functions on $\Omega, 1 \leq p<\infty$ (see [6]). Recall (from [6]) that if $1<p<\infty$ and $1 / p+1 / q=1$, the space $L^{q}\left(\mu, Z^{*}\right)$ is isometrically embedded in $L^{p}(\mu, Z)^{*}$ and they coincide if and only if $Z^{*}$ has the Radon-Nikodým Property (RNP) with respect to $\mu$.

We note the following 
Proposition 1. Let $X=L^{p}(\mu, Z)$ and $F=L^{q}\left(\mu, Z^{*}\right), 1<p<\infty$, $1 / p+1 / q=1$. Then every simple function in $S(X)$ is in $T$. So, $T$ is dense in $S(X)$. Moreover, the pair $(X, F)$ satisfies $(* *)$.

Proof. Let $x=\sum_{i=1}^{n} x_{i} \chi_{E_{i}}$ be any simple function and let $\phi: S(Z) \rightarrow$ $S\left(Z^{*}\right)$ be any support mapping. Define

$$
\Phi(x)=\sum_{i=1}^{n}\left\|x_{i}\right\|^{p-1} \phi\left(\frac{x_{i}^{*}}{\left\|x_{i}^{*}\right\|}\right) \chi_{E_{i}} .
$$

Then $\Phi(x) \in D(x) \cap S(F)$. This proves the first part of the proposition.

Now, let $x \in S\left(L^{p}(Z)\right), f \in S\left(L^{q}\left(Z^{*}\right)\right)$ and $\varepsilon>0$ be such that $f(x)>$ $1-\varepsilon^{2}$. Choose $0<\eta<\varepsilon$ such that $0<\eta[2(\varepsilon+1)-\eta]<f(x)-\left(1-\varepsilon^{2}\right)$. Let $z$ and $g$ be simple functions in $S\left(L^{p}(Z)\right)$ and $S\left(L^{q}\left(Z^{*}\right)\right)$ respectively such that $\|x-z\|_{p}<\eta$ and $\|f-g\|_{q}<\eta$. Refining the partitions if necessary, we may assume that there is a finite partition $\left\{E_{1}, \ldots, E_{n}\right\}$ of $\Omega$ such that $z=\sum_{i=1}^{n} z_{i} \chi_{E_{i}}$ and $g=\sum_{i=1}^{n} z_{i}^{*} \chi_{E_{i}}$ where $z_{i} \in Z, z_{i}^{*} \in Z^{*}$ and $\chi_{A}$ denotes the indicator function of $A$. Hence, $g(z)=\sum_{i=1}^{n} z_{i}^{*}\left(z_{i}\right) \mu\left(E_{i}\right)>$ $f(x)-2 \eta>1-(\varepsilon-\eta)^{2}$, by the choice of $\eta$. Now, consider the discrete measure space $\Omega^{\prime}=\{1, \ldots, n\}$ with measure $P$, where $P(i)=\mu\left(E_{i}\right)$. Then $z$ and $g$ can be isometrically identified with elements of $S\left(L^{p}(P, Z)\right)$ and $S\left(L^{q}\left(P, Z^{*}\right)\right)$ respectively. But as $P$ is discrete, $L^{p}(P, Z)^{*}=L^{q}\left(P, Z^{*}\right)$ and so $(* *)$ is satisfied, i.e, there exist vectors $\left(y_{1}, \ldots, y_{n}\right)$ and $\left(y_{1}^{*}, \ldots, y_{n}^{*}\right)$ in $S\left(L^{p}(P, Z)\right)$ and $S\left(L^{q}\left(P, Z^{*}\right)\right)$ respectively such that $\sum_{i=1}^{n} y_{i}^{*}\left(y_{i}\right) P(i)=1$ and $\left[\sum_{i=1}^{n}\left\|z_{i}-y_{i}\right\|^{p} P(i)\right]^{1 / p} \leq \varepsilon-\eta$ and $\left[\sum_{i=1}^{n}\left\|z_{i}^{*}-y_{i}^{*}\right\|^{q} P(i)\right]^{1 / q} \leq \varepsilon-\eta$. Put $y=\sum_{i=1}^{n} y_{i} \chi_{E_{i}}$ and $f_{y}=\sum_{i=1}^{n} y_{i}^{*} \chi_{E_{i}}$. Then $y \in S\left(L^{p}(\mu, Z)\right), f_{y} \in$ $S\left(L^{q}\left(\mu, Z^{*}\right)\right)$ and $f_{y}(y)=1$. Further, $\|x-y\|_{p} \leq(\varepsilon-\eta)+\eta=\varepsilon$ and $\left\|f-f_{y}\right\|_{q} \leq \varepsilon$.

Here we have

THEOREM 3. For any Banach space $Z$, any finite measure space $(\Omega, \Sigma, \mu)$ and any $1<p<\infty$, the following are equivalent:

(i) $Z$ has the MIP.

(ii) $L^{p}(\mu, Z)$ has the $L^{q}\left(\mu, Z^{*}\right)-M I P$.

Proof. The proof of this theorem is already essentially contained in the proof of Theorem 8 in [1]. One only has to use $(\mathrm{a}) \Leftrightarrow(\mathrm{d}) \Leftrightarrow(\mathrm{f})$ of Theorem 2 above, instead of the corresponding equivalence for the MIP (Theorem 2.1 of $[9])$.

Rem arks. 1. Theorem 8 of [1] clearly follows from Theorem 3 .

2. As noted in [1], if the MIP implies that the space is Asplund, the MIP should lift to the Bochner $L^{p}$-spaces and that would also prove that the space is indeed Asplund. But the above theorem indicates the difficulties inherent in this approach. 
Acknowledgement. The author is indebted to his supervisor, Prof.

A. K. Roy, for his continuous encouragement and help in the present work.

\section{REFERENCES}

[1] P. Bandyopadhyaya and A. K. Roy, Some stability results for Banach spaces with the Mazur Intersection Property, Indag. Math. 1 (2) (1990), 137-154.

[2] E. Bishop and R. R. Phelps, A proof that every Banach space is subreflexive, Bull. Amer. Math. Soc. 67 (1961), 97-98.

[3] B. Bollobás, An extension to the theorem of Bishop and Phelps, Bull. London Math. Soc. 2 (1970), 181-182.

[4] R. D. Bourgin, Geometric Aspects of Convex Sets with the Radon-Nikodym Property, Lecture Notes in Math. 993, Springer, 1983.

[5] G. Choquet, Lectures on Analysis, Vol. II, W. A. Benjamin, New York 1969.

[6] J. Diestel and J. J. Uhl, Jr., Vector Measures, Math. Surveys 15, Amer. Math. Soc., Providence, R.I., 1977.

[7] N. Dunford and J. T. Schwartz, Linear Operators, Vol. I, Interscience, New York 1958.

[8] J. R. Giles, Convex Analysis with Application in the Differentiation of Convex Functions, Pitman Adv. Publ. Program, Boston 1982.

[9] J. R. Giles, D. A. Gregory and B. Sims, Characterization of normed linear spaces with Mazur's intersection property, Bull. Austral. Math. Soc. 18 (1978), 105-123.

[10] S. Mazur, Über schwache Konvergenz in den Räumen $\left(L^{p}\right)$, Studia Math. 4 (1933), $128-133$.

[11] R. R. Phelps, A representation theorem for bounded convex sets, Proc. Amer. Math. Soc. 11 (1960), 976-983.

[12] A. Sersouri, The Mazur property for compact sets, Pacific J. Math. 133 (1988), $185-195$.

[13] —, Mazur's intersection property for finite dimensional sets, Math. Ann. 283 (1989), $165-170$.

[14] J. H. M. Whitfield and V. Zizler, Mazur's intersection property of balls for compact convex sets, Bull. Austral. Math. Soc. 35 (1987), 267-274.

[15] V. Zizler, Note on separation of convex sets, Czechoslovak Math. J. 21 (1971), 340-343.

[16] - Renorming concerning Mazur's intersection property of balls for weakly compact convex sets, Math. Ann. 276 (1986), 61-66.

DIVISION OF THEORETICAL STATISTICS AND MATHEMATICS

INDIAN STATISTICAL INSTITUTE

203 BARRACKPORE TRUNK ROAD

CALCUTTA 700 035, INDIA 